\title{
SELF-DUAL MODULES IN CHARACTERISTIC TWO AND NORMAL SUBGROUPS
}

\author{
ROD GOW AND JOHN MURRAY
}

\begin{abstract}
We prove Clifford theoretic results which only hold in characteristic 2 .
Let $G$ be a finite group, let $N$ be a normal subgroup of $G$ and let $\varphi$ be an irreducible 2 -Brauer character of $N$. We show that $\varphi$ occurs with odd multiplicity in the restriction of some self-dual irreducible Brauer character $\theta$ of $G$ if and only if $\varphi$ is $G$-conjugate to its dual. Moreover, if $\varphi$ is self-dual then $\theta$ is unique and the multiplicity is 1 .

Next suppose that $\theta$ is a self-dual irreducible 2-Brauer character of $G$ which is not of quadratic type. We prove that the restriction of $\theta$ to $N$ is a sum of distinct self-dual irreducible Brauer character of $N$, none of which have quadratic type. Moreover, $G$ has no self-dual irreducible 2-Brauer character of non-quadratic type if and only if $N$ and $G / N$ satisfy the same property.

Finally, suppose that $b$ is a real 2-block of $N$. We show that there is a unique real 2-block of $G$ covering $b$ which is weakly regular with respect to $N$.
\end{abstract}

\section{Statement of RESUlts}

Throughout the paper $G$ is a finite group and $N$ is a normal subgroup of $G$. We fix a 2 -modular system $(K, R, F)$ for $G$. So $R$ is a complete discrete valuation ring which has field of fractions $K$ of characteristic 0 and residue field $F=R / J(R)$ of characteristic 2. We will assume that $K$ and $F$ are splitting fields for all subgroups of $G$. For example, this holds if $K$ contains a primitive $|G|$-th root of unity. We use $r^{*}$ to denote the image of $r \in R$ in $F$. Each integer $m$ can be factored as $m=m_{2} m_{2^{\prime}}$, where $m_{2}$ is a power of 2 and $m_{2^{\prime}}$ is odd.

We use $\operatorname{Irr}(G)$ to denote the irreducible $K$-characters of $G$. These have values in a cyclotomic subfield of $K$ which can be identified with a subfield of $\mathbb{C}$. So $\operatorname{Irr}(G)$ can be identified with the irreducible complex characters of $G$. Next recall that the Brauer character of an $F G$-module is an $R$-valued class function defined on the 2-regular (odd order) elements of $G$. The Brauer characters of the irreducible $F G$-modules are called the irreducible 2-Brauer characters of $G$. We use $\operatorname{IBr}(G)$ to denote all such characters. The dual of a character $\theta$ is the character $\bar{\theta}$ defined by $\bar{\theta}(g):=\theta\left(g^{-1}\right)$, for all $g \in G$. We say that $\theta$ is self-dual if $\theta=\bar{\theta}$. This holds if and only if $\theta$ is the character of some self-dual module.

Let $\theta$ be an irreducible Brauer character of $G$ and let $\varphi$ be an irreducible Brauer character of a subgroup $H$ of $G$. We say that $\theta$ lies over $\varphi$ if $\theta$ is constituent of the induced

Date: August 19, 2020. 
character $\varphi \uparrow^{G}$. Likewise we say that $\varphi$ lies under $\theta$ if $\varphi$ is a constituent of the restricted character $\theta \downarrow_{H}$. There is no analogue of Frobenius reciprocity for Brauer characters. So the fact that $\theta$ lies over $\varphi$ does not imply that $\varphi$ lies under $\theta$, and conversely. However these implications do hold if $H$ is normal in $G$ [N, p155 and (8.7)]. Our first result is:

Theorem 1. Let $\varphi$ be an irreducible 2-Brauer character of $N$. Then $\varphi$ lies under some self-dual irreducible 2-Brauer character $\theta$ of $G$ if only if $\varphi$ is $G$-conjugate to $\bar{\varphi}$. If such a self-dual $\theta$ exists, it can be chosen so that $\varphi$ occurs with odd multiplicity in $\theta \downarrow_{N}$.

Our second result is:

Theorem 2. Let $\varphi$ be a self-dual irreducible 2-Brauer character of $N$. Then

(i) $\varphi$ extends to its stabilizer in $G$, and exactly one of these extensions is self-dual.

(ii) $G$ has a unique self-dual irreducible 2 -Brauer character $\theta$ such that $\varphi$ occurs with odd multiplicity in $\theta \downarrow_{N}$.

(iii) $\varphi$ occurs with multiplicity 1 in $\theta \downarrow_{N}$.

We will refer to $\theta$ as the canonical irreducible Brauer character of $G$ lying over $\varphi$. The module form of this theorem is stated and proved in Theorem 9 below.

Replacing the normal subgroup $N$ by a subnormal subgroup $H$, part (ii) of Theorem 2 still holds. However part (iii) need not hold. Indeed the multiplicity can be arbitrarily large, as J. Müller has pointed out. We outline his example at the end of Section 3 .

Theorem 2 is similar in flavour to a result of I. M. Richards. In $[\mathrm{R}]$ he proved that when $G / N$ has odd order, each self-dual irreducible $K$-character of $N$ extends to its stabilizer in $G$, and has a unique self-dual extension.

Example: Let $G$ be the semi-dihedral group of order $2^{n} \geq 16$, and let $N$ be the centre of $G$. Then $N$ has a unique, hence self-dual, non-trivial irreducible $K$-character. However all faithful irreducible $K$-characters of $G$ have degree 2 and none of them is self-dual. So neither Theorems 1 nor 2 generalize to irreducible $K$-characters nor to irreducible $p$-Brauer characters, for primes $p \neq 2$.

Next recall that a non-trivial irreducible 2-Brauer character of $G$ is said to have quadratic type if the corresponding $F G$-module affords a non-zero $G$-invariant quadratic form. Our first application is:

Theorem 3. Let $\theta$ be a non-quadratic type self-dual irreducible 2-Brauer character of $G$ which does not lie over the trivial character of $N$. Then $\theta \downarrow_{N}$ is a sum of non-quadratic type self-dual irreducible Brauer characters of $N$, each occurring with multiplicity 1.

The second application is to blocks. For undefined notation, see Section 6 below and for a full exposition of block theory, see Chapter 5 of [NT].

Let $B$ be a 2-block of irreducible $K$-characters of $G$. Then the duals of the characters in $B$ form another 2-block $B^{\circ}$, called the contragredient of $B$. We say that $B$ is real if $B=B^{o}$. Recall that $B$ is said to cover a 2-block $b$ of $N$ if the restriction of an irreducible character in $B$ contains an irreducible character in $b$, and that $B$ is said to be weakly regular (with respect to $N$ ) if it has maximal defect among the blocks of $G$ which cover $b$. 
Theorem 4. Let $b$ be a 2-block of $N$. Then

(i) $G$ has a real weakly regular 2-block covering $b$ if and only if $b$ is $G$-conjugate to $b^{o}$.

(ii) $G$ has a unique real weakly regular 2-block covering $b$ if $b=b^{o}$.

We prove (i) in Lemma 18 and (ii) in Lemma 20.

Recall that corresponding to each irreducible 2-Brauer character $\theta, G$ has a principal indecomposable character $\Phi_{\theta}$. Then $\Phi_{\theta}$ is a $K$-character of $G$ which vanishes off the 2regular elements of $G$. We use the following result, which is implicit in [GW93, 1.4], to prove Theorem 4. As it may be of independent interest, we include a short proof here:

Lemma 5. Let $B$ be a 2-block of $G$. Then $B$ has an odd number of height 0 irreducible Brauer characters $\theta$ such that $\Phi_{\theta}(1)_{2}=|G|_{2}$.

Proof. Let $D$ be a defect group of $B$. Then Brauer showed that $\frac{\operatorname{dim}(B)}{|G||G: D|}$ is a unit in $R$. See [NT, 5.10.1]. Now $\operatorname{dim}(B)=\sum_{\theta \in \operatorname{IBr}(b)} \Phi_{\theta}(1) \theta(1)$. It is known that $\Phi_{\theta}(1) /|G|$ and $\theta(1) /|G: D|$ belong to $R$, for all $\theta \in \operatorname{IBr}(B)$. So Brauer's result gives us an identity in $F$ :

$$
\sum_{\theta \in \operatorname{IBr}(B)}\left(\frac{\Phi_{\theta}(1)}{|G|}\right)^{*}\left(\frac{\theta(1)}{|G: D|}\right)^{*}=\left(\frac{\operatorname{dim}(B)}{|G||G: D|}\right)^{*}=1_{F} .
$$

The contribution of $\theta$ to the left hand side is $1_{F}$, if $\theta$ has height 0 and $\Phi_{\theta}(1)_{2}=|G|_{2}$. Otherwise the contribution is $0_{F}$. So the lemma follows directly from the above equality.

Many 2-blocks have an odd number of height 0 irreducible Brauer characters. For example, the main result of [KOW] is that each 2-block of a symmetric group has a unique height 0 irreducible Brauer character. Furthermore, it is known that each principal indecomposable character $\Phi$ of a finite solvable group satisfies $\Phi(1)_{2}=|G|_{2}$. So Lemma 5 implies that each 2-block of a finite solvable group has an odd number of height 0 irreducible Brauer characters. However, as B. Sambale has pointed out, the faithful 2block of 3.Suz.2 has four height 0 irreducible Brauer characters. Three of these satisfy the condition of Lemma 5 on their principal indecomposable character degree. T. Wada and J. Müller have independently noted this example, and the fact that it is unique in the [ModAtlas] database.

\section{ReAl ORBItS OF IRREDUCIBLE BRAUER CHARACTERS}

Recall that $g \in G$ is said to be real in $G$ if $x g x^{-1}=g^{-1}$, for some $x \in G$. Similarly a conjugacy class of $G$ is real if its elements are real, and 2-regular if its elements have odd order. Now $G$ acts on the conjugacy classes, the irreducible $K$-characters and the Brauer characters of its normal subgroup $N$. We say that a $G$-orbit of conjugacy classes of $N$ is real if its union is a real conjugacy class of $G$. Likewise we say that a $G$-orbit of irreducible Brauer characters of $N$ is real if it contains the duals of its characters. 
Proof of Theorem 1. It is clear that each self-dual irreducible Brauer character of $G$ lies over a real $G$-orbit of irreducible Brauer characters of $N$.

Suppose that $G$ has $\ell$ conjugacy classes of 2-regular elements, with representatives $g_{1}, \ldots, g_{\ell}$. Let $\theta_{1}, \ldots, \theta_{\ell}$ be the irreducible Brauer characters of $G$ and let $\Phi_{1}, \ldots \Phi_{\ell}$ be the corresponding principal indecomposable characters of $G$. The second orthogonality relations give

$$
\sum_{\chi \in \operatorname{Irr}(G)} \chi\left(g_{i}^{-1}\right) \chi\left(g_{j}\right)=\delta_{i, j}\left|\mathrm{C}_{G}\left(g_{i}\right)\right|, \quad \text { for all } i, j \in\{1, \ldots, \ell\} .
$$

Now for all $\chi \in \operatorname{Irr}(G)$, we have $\chi\left(g_{j}\right)=\sum_{u=1}^{\ell} d_{\chi, \theta_{u}} \theta_{u}\left(g_{j}\right)$, where the $d_{\chi, \theta_{u}}$ are nonnegative integers, called decomposition numbers. Then for all $u=1, \ldots, \ell$, we have $\Phi_{u}\left(g_{i}\right)=\sum_{\chi \in \operatorname{Irr}(G)} d_{\chi, \theta_{u}} \chi\left(g_{i}\right)$. It is known that $\frac{\Phi_{u}\left(g_{i}^{-1}\right)}{\left|\mathrm{C}_{G}\left(g_{i}\right)\right|} \in R$, for all $u, i$. So the above displayed equation can be rewritten in $R$ as

$$
\sum_{u=1}^{\ell} \frac{\Phi_{u}\left(g_{i}^{-1}\right)}{\left|\mathrm{C}_{G}\left(g_{i}\right)\right|} \theta_{u}\left(g_{j}\right)=\delta_{i, j}, \quad \text { for all } i, j \in\{1, \ldots, \ell\} .
$$

In particular the Brauer character table $\left[\theta_{i}\left(g_{j}\right)\right]$ of $G$ is a non-singular $\ell \times \ell$ matrix. (We note that the proof of $[\mathrm{N},(2.18)]$ shows that $\operatorname{det}\left[\theta_{i}\left(g_{j}\right)\right]^{2}= \pm \prod_{j=1}^{\ell}\left|\mathrm{C}_{G}\left(g_{j}\right)\right|_{2^{\prime}}$.)

Suppose that $G$ has $r$ real conjugacy classes of 2-regular elements, which we may assume have representatives $g_{1}, \ldots, g_{r}$. We choose notation so that $\theta_{1}, \ldots, \theta_{r}$ are the self-dual irreducible Brauer characters of $G$. Then the self-dual Brauer character table of $G$ is the $r \times r$ submatrix $T:=\left[\theta_{i}\left(g_{j}\right)\right]$ of the Brauer character table. Suppose that $u \in$ $\{r+1, \ldots, \ell\}$. Then there is a unique $\bar{u} \in\{r+1, \ldots, \ell\}$, with $\bar{u} \neq u$, such that $\theta_{\bar{u}}=\bar{\theta}_{u}$. So $\frac{\Phi_{u}\left(g_{i}^{-1}\right)}{\left|\mathrm{C}_{G}\left(g_{i}\right)\right|} \theta_{u}\left(g_{j}\right)=\frac{\Phi_{\bar{u}}\left(g_{i}^{-1}\right)}{\left|\mathrm{C}_{G}\left(g_{i}\right)\right|} \theta_{\bar{u}}\left(g_{j}\right)$, for all $i, j$. So the contribution of the summands indexed by $u$ and $\bar{u}$ to (10) is $0 \bmod J(R)$, and we deduce that

$$
\sum_{u=1}^{r} \frac{\Phi_{u}\left(g_{i}^{-1}\right)}{\left|\mathrm{C}_{G}\left(g_{i}\right)\right|} \theta_{u}\left(g_{j}\right) \equiv \delta_{i, j} \quad \bmod J(R), \quad \text { for all } i, j \in\{1, \ldots, r\} .
$$

As $R$ is a local ring, it follows that $T$ is invertible, with inverse congruent to the $r \times r$ matrix $\left[\frac{\Phi_{i}\left(g_{j}^{-1}\right)}{\left|\mathrm{C}_{G}\left(g_{j}\right)\right|}\right] \bmod J(R)$. In particular $\operatorname{det}(T) \notin J(R)$.

Now suppose that $G$ has $t$ real conjugacy classes of 2-regular elements which are contained in $N$, with representatives $n_{1}, \ldots, n_{t}$. We relabel the $\theta_{1}, \ldots, \theta_{r}$ so that the $t \times t$ submatrix $S:=\left[\theta_{i}\left(n_{j}\right)\right]$ of $T$ satisfies $\operatorname{det}(S) \notin J(R)$.

For $i=1, \ldots, t$, let $\varphi_{i}$ be an irreducible Brauer character of $N$ which is a constituent of $\theta_{i} \downarrow_{N}$, and set $\hat{\varphi}_{i}$ as the sum of the distinct $G$-conjugates of $\varphi_{i}$. Then $\theta_{i} \downarrow_{N}=e_{i} \hat{\varphi}_{i}$, for some positive integer $e_{i}$. The non-singularity of $S$ implies that all the multiplicities $e_{1}, \ldots, e_{t}$ are odd and $\varphi_{1}, \ldots, \varphi_{t}$ lie in distinct $G$-orbits. Moreover each of these orbits is real, as each $\theta_{i}$ is self-dual. 
By the non-singularity of the Brauer character table of $N$ and Brauer's permutation lemma, $G$ has $t$ real orbits on the irreducible Brauer characters of $N$. So $\varphi_{1}, \ldots, \varphi_{t}$ represent all real $G$-orbits of irreducible Brauer characters of $N$.

Our work above shows that if $\varphi$ is an irreducible Brauer character of $N$ which is $G$ conjugate to $\bar{\varphi}$, then $G$ has a self-dual irreducible Brauer character $\theta$ such that $\varphi$ occurs with odd multiplicity in $\theta \downarrow_{N}$. This concludes the proof.

\section{Clifford Theory For SElF-DUAL IRREDUCiBle MOdUleS}

We prove Theorem 2 in this section. Recall that the dual of an $F G$-module $V$ is the $F G$-module $V^{*}:=\operatorname{Hom}_{F}(V, F)$. We say that $V$ is self-dual if $V \cong V^{*}$ as $F G$-modules. For the reader's convenience, we begin by stating a module version of Clifford's Theorem:

Lemma 6 (Clifford 1937). Let $F$ be an arbitary field, let $V$ be an irreducible FG-module and let $W$ be an irreducible submodule of $V \downarrow_{N}$. Set $T$ as the stabilizer of $W$ in $G$. Then

(i) $V \downarrow_{N} \cong e\left(W_{1} \oplus \cdots \oplus W_{n}\right)$ for some integer $e>0$, where $W_{1}, \ldots, W_{n}$ are the distinct $G$-conjugates of $W$. In particular $V \downarrow_{N}$ is semi-simple.

(ii) Let $U$ be the sum of all submodules of $V \downarrow_{N}$ which are isomorphic to $W$. Then $U$ is an irreducible FT-module, $U \downarrow_{N}=e W$ and $U \uparrow G=V$.

(iii) If $W$ is absolutely irreducible, it extends to a projective FT-module $\hat{W}$ and $U \cong$ $P \otimes \hat{W}$, for some projective $F(T / N)$-module $P$.

(iv) If $W$ extends to an FT-module $\hat{W}$, then the distinct irreducible $F G$-modules lying over $W$ are $\left(S_{1} \otimes \hat{W}\right) \uparrow^{G}, \ldots,\left(S_{t} \otimes \hat{W}\right) \uparrow G$ where $S_{1}, \ldots, S_{t}$ are the distinct irreducible $F(T / N)$-modules.

Proof. See for example Huppert and Blackburn, Finite Groups II, VII, 9.12.

Next we point out that the following result, generally known as Fong's Lemma, holds for an arbitrary field $F$ of characteristic 2. In particular $F$ need not be perfect:

Lemma 7. Let $G$ be a finite group, let $F$ be an arbitrary field of characteristic 2 and let $V$ be a non-trivial self-dual irreducible FG-module. Then $V$ affords a non-degenerate $G$-invariant alternating bilinear form. In particular $\operatorname{dim}(V)$ is even.

Proof. The self-duality of $V$ is equivalent to the existence of a non-degenerate $G$-invariant bilinear form $B: V \times V \rightarrow F$. If $B$ is not symmetric, set $\hat{B}\left(v_{1}, v_{2}\right)=B\left(v_{1}, v_{2}\right)+B\left(v_{2}, v_{1}\right)$, for all $v_{1}, v_{2} \in V$. Then $\hat{B}$ is a $G$-invariant non-zero symmetric bilinear form on $V$. Now $\operatorname{rad}(\hat{B})$ is a submodule of $V$ and $V$ is irreducible. So $\operatorname{rad}(\hat{B})=0$ and $\hat{B}$ is non-degenerate.

The first paragraph shows that $V$ affords a $G$-invariant non-degenerate symmetric bilinear form, henceforth denoted $B$. We claim that $B$ is alternating, meaning $B(v, v)=0$, for all $v \in V$. For suppose otherwise. Set $Q(v):=B(v, v)$, for all $v \in V$. Then $Q$ is a non-zero $G$-invariant quadratic form on $V$. Now $Q$ is additive, as for all $v_{1}, v_{2} \in V$, we 
have

$$
\begin{aligned}
Q\left(v_{1}+v_{2}\right) & =B\left(v_{1}+v_{2}, v_{1}+v_{2}\right) \\
& =B\left(v_{1}, v_{1}\right)+B\left(v_{1}, v_{2}\right)+B\left(v_{2}, v_{1}\right)+B\left(v_{2}, v_{2}\right) \\
& =Q\left(v_{1}\right)+Q\left(v_{2}\right), \quad \text { using } B\left(v_{1}, v_{2}\right)+B\left(v_{2}, v_{1}\right)=0 .
\end{aligned}
$$

Moreover $Q(\lambda v)=\lambda^{2} Q(v)$, for all $\lambda \in F$ and $v \in V$. Define $U:=\{v \in V \mid Q(v)=0\}$. Then our work shows that $U$ is a submodule of $V$. But $U \neq V$, as $Q \neq 0$. So $U=0$, by irreducibility of $V$. Let $v \in V$ and $g \in G$. Then $Q(g v+v)=Q(g v)+Q(v)=0$, as $Q$ is additive and $G$-invariant. So $g v+v \in U$, whence $g v=v$. But then $G$ acts trivially on $V$, contrary to hypothesis. This proves our claim.

The final statement follows as every symplectic vector space has even dimension.

Corollary 8. Let $G$ be a finite group and let $F$ be an arbitrary field of characteristic 2 . Then the radical of $F G$ has odd codimension in $F G$.

Proof. We use $\operatorname{rad}(F G)$ to denote the radical of $F G$, which is the intersection of the annihilators of all irreducible $F G$-modules. Suppose first that $F$ is a splitting field for $G$. Let $\theta_{1}, \ldots, \theta_{\ell}$ be the irreducible Brauer characters of $G$, with $\theta_{1}, \ldots, \theta_{r}$ precisely the self-dual characters, and $\theta_{1}$ the trivial Brauer character. We have

$$
\operatorname{dim}(F G)-\operatorname{dim}(\operatorname{rad}(F G))=\theta_{1}(1)^{2}+\cdots+\theta_{\ell}(1)^{2} .
$$

Now $\theta_{1}(1)=1$ and $\theta_{i}(1)$ is even for $2 \leq i \leq r$, by Fong's Lemma. If $i>r$, we may pair $\theta_{i}$ with its dual, and these two characters have the same degree. It is now clear that

$$
\theta_{1}(1)^{2}+\cdots+\theta_{\ell}(1)^{2}
$$

is odd, and the result follows in this case.

Now suppose that $F$ is any field of characteristic 2. Set $E=F(\omega)$, where $\omega$ is a primitive $|G|_{2^{\prime}}$-th root of unity in an extension field of $F$. Then $E$ is a splitting field for $G$ and a finite separable extension of $F$. As $F G$ contains an $E$-basis of $E G$, it is a standard fact that $\operatorname{rad}(E G)$ is the $E$-span of $\operatorname{rad}(F G)$. In particular $\operatorname{dim}_{E}(\operatorname{rad}(E G))=\operatorname{dim}_{F}(\operatorname{rad}(F G))$. The

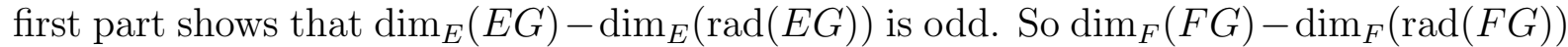
is odd in this case also.

For the rest of this section $F$ is a perfect field of characteristic 2. Here is the module version of Theorem 2 :

Theorem 9. Let $W$ be a self-dual irreducible FN-module. Then $W$ extends to its stabilizer in $G$, and there is a unique extension $\hat{W}$ which is self-dual.

Set $V:=\hat{W}^{\uparrow} G$. Then $V$ is a self-dual irreducible $F G$-module, and $V \downarrow_{N} \cong W_{1} \oplus \cdots \oplus W_{n}$, where $W_{1}, \ldots, W_{n}$ are the distinct $G$-conjugates of $W$. Moreover $V$ is the unique self-dual irreducible FG-module such that $W$ occurs with odd multiplicity in $V \downarrow_{N}$.

Proof. We may assume that $W$ is non-trivial and $G$-invariant. As $W$ is a self-dual $F N$ module, it affords a non-degenerate $N$-invariant bilinear form $B: W \times W \rightarrow F$. An application of Schur's Lemma shows that $B$ is unique up to scaling. 
Let $X: N \rightarrow \mathrm{GL}(W)$ be the $F$-representation given by $W$. For each $g \in G$, we define the conjugate representation $X^{g}$ of $N$ by

$$
X^{g}(n)=X\left(g n g^{-1}\right), \quad \text { for all } n \in N .
$$

Then $X$ and $X^{g}$ are equivalent representations, as $W$ is $G$-invariant. So there is $Y(g) \in$ $\mathrm{GL}(W)$ such that

$$
Y(g) X(n)=X^{g}(n) Y(g), \quad \text { for all } n \in N .
$$

We choose $Y(g)=X(g)$, whenever $g \in N$. Now for all $g, h \in G$, we have

$$
\left[Y(g h)^{-1} Y(g) Y(h)\right] X(n)=X(n)\left[Y(g h)^{-1} Y(g) Y(h)\right] .
$$

So by Schur's Lemma there is a non-zero $\alpha(g, h) \in F$ such that

$$
Y(g h)=\alpha(g, h) Y(g) Y(h) .
$$

Then $\alpha: G \times G \rightarrow F^{\times}$is an $F$-valued cocycle and $Y$ is a projective representation of $G$ which extends $X$.

Next, for all $g \in G$, we define the bilinear form $B^{g}: W \times W \rightarrow F$ by

$$
B^{g}(u, v)=B(Y(g) u, Y(g) v), \quad \text { for all } u, v \in W .
$$

Then for all $n \in N$ we have

$$
\begin{aligned}
B^{g}(X(n) u, X(n) v) & =B(Y(g) X(n) u, Y(g) X(n) v) \\
& =B\left(X\left(g n g^{-1}\right) Y(g) u, X\left(g n g^{-1}\right) Y(g) v\right) \\
& =B(Y(g) u, Y(g) v), \quad \text { as } B \text { is } X \text {-invariant } \\
& =B^{g}(u, v) .
\end{aligned}
$$

This shows that $B^{g}$ is $X$-invariant. As $B$ is unique up to scalars

$$
B^{g}=\lambda(g) B, \quad \text { for some } \lambda(g) \in F^{\times} .
$$

As $B$ is $N$-invariant, we have $\lambda(n)=1$, for all $n \in N$.

Now for all $g, h \in G$ we have $B^{g h}=\lambda(g h) B$. On the other hand

$$
\begin{aligned}
B^{g h}(u, v) & =B(Y(g h) u, Y(g h) v) \\
& =B(\alpha(g, h) Y(g) Y(h) u, \alpha(g, h) Y(g) Y(h) v), \quad \text { by (2), } \\
& \left.=\alpha(g, h)^{2} \lambda(g) \lambda(h) B(u, v), \quad \text { by (3) }\right) .
\end{aligned}
$$

Comparing these expressions, we see that

$$
\lambda(g h)=\alpha(g, h)^{2} \lambda(g) \lambda(h) .
$$

Since $F$ is perfect, for each $g$ in $G$, there exists $\mu(g) \in F$ such that $\mu(g)^{2}=\lambda(g)$. Set $\hat{Y}(g)=\mu(g)^{-1} Y(g)$ for all $g \in G$. Then $\hat{Y}$ is a projective representation of $G$ which extends $X$. Moreover $\hat{Y}$ corresponds to the cocycle $\beta(g, h):=\mu(g) \mu(h) \mu(g h)^{-1} \alpha(g, h)$.

Now $\beta(g, h)^{2}=\lambda(g) \lambda(h) \lambda(g h)^{-1} \alpha(g, h)^{2}=1$ and $\operatorname{char}(F)=2$. So $\beta(g, h)=1$, for all $g, h \in G$. This means that $\hat{Y}$ is an $F$-representation of $G$ which extends $X$. 
If we now consider the action of the elements $\hat{Y}(g)$ on the bilinear form $B$, a repetition of an earlier argument shows that for all $u$ and $v$ in $W$, and all $g \in G$,

$$
B(\hat{Y}(g) u, \hat{Y}(g) v)=\epsilon(g) B(u, v)
$$

for some nonzero scalar $\epsilon(g)$. The fact that $\hat{Y}$ is a representation of $G$, and $B$ is $N$ invariant now implies that $\epsilon$ is a homomorphism $G / N \rightarrow F^{\times}$.

Finally, as $F$ has characteristic $2, \epsilon$ has odd order in the character group of $G / N$. So as $F$ is perfect, $\epsilon=\delta^{2}$ for a unique homomorphism $\delta: G / N \rightarrow F^{\times}$. Then if we set $\hat{X}(g)=\delta(g)^{-1} \hat{Y}(g)$, we find that $\hat{X}$ is also an $F$-representation of $G$ which extends $X$. Moreover $\hat{X}$ is self-dual, as we can easily check that it leaves $B$ invariant.

Let $\hat{W}$ be the irreducible self-dual $F G$-module corresponding to $\hat{X}$. So $\hat{W}$ extends $W$. Then $S \otimes \hat{W}$ give all irreducible $F G$-modules lying over $W$, as $S$ ranges over all irreducible $F G / N$-modules. Recall that $S \otimes \hat{W} \cong S^{\prime} \otimes \hat{W}$ if and only if $S \cong S^{\prime}$. So $S \otimes \hat{W}$ is self-dual if and only if $S$ is self-dual. Fong's Lemma implies that $\operatorname{dim}(S \otimes \hat{W})$ is an even multiple of $\operatorname{dim}(\hat{W})$, if $S$ is non-trivial and self-dual. So $\hat{W}$ is the unique extension of $W$ to $G$ which is self-dual. The statements about $V$ are now consequences of Lemma 6 ,

We will refer to $V$ as the canonical self-dual irreducible $F G$-module lying over $W$. Our Corollary, which is probably known, is an analogue of Richards' Theorem [R] for irreducible 2-Brauer characters:

Corollary 10. Suppose that $|G: N|$ is odd. Then

(i) If $W$ is a self-dual irreducible $F N$-module, then $W \uparrow G$ has a unique self-dual composition factor, up to isomorphism.

(ii) If $V$ is a self-dual irreducible $F G$-module then $V \downarrow_{N}$ is a sum of distinct self-dual irreducible $F N$-modules.

In particular induction-restriction defines a natural correspondence between the self-dual irreducible FG-modules and the G-orbits of self-dual irreducible FN-modules.

Proof. We may assume that $W$ is $G$-invariant. Let $\hat{W}$ be the unique self-dual irreducible $F G$-module which extends $W$. Then all irreducible $F G$-modules lying over $W$ have the form $U \otimes W$, for some irreducible $F G / N$-module $U$. But $G / N$ has odd order. So $U$ is self-dual if and only if it is trivial. It follows that $\hat{W}$ is the unique self-dual irreducible $F G$-module lying over $W$. All composition factors of $W \uparrow G$ lie over $W$. So (i) holds.

For (ii), write $V \downarrow_{N}=e\left(W_{1} \oplus \cdots \oplus W_{t}\right)$, where $e, t \geq 1$ and $W_{1}, \ldots, W_{t}$ are distinct irreducible $F N$-modules. Now $V \downarrow_{N}$ is a self-dual irreducible $F N$-module. So for each $i=1, \ldots, t, W_{i}^{*} \cong W_{j}$, for some $j=1, \ldots, t$. The map $i \mapsto j$ is an involutary permutation of $\{1, \ldots, t\}$. But $t$ is odd, as by Clifford Theory it divides $|G: N|$. So there exists $i$ such that $W_{i}^{*} \cong W_{i}$, whence all $W_{1}, \ldots, W_{t}$ are self-dual. Now by part (i), $V$ is the unique self-dual irreducible $F G$-module lying over $W_{1}$. It then follows from Theorem 9 that $e=1$ i.e. $V \downarrow_{N}=W_{1} \oplus \cdots \oplus W_{t}$.

The last statement follows from (i) and (ii). 
We note that Lemma [ can be used to show that the restriction of an irreducible $F G$ module to a subnormal subgroup of $G$ is semi-simple. Part (ii) of Theorem 2 extends to subnormal subgroups:

Corollary 11. Let $H$ be a subnormal subgroup of $G$ and let $U$ be a self-dual irreducible $F H$-module. Then there is a unique self-dual irreducible FG-module $V$ such that $U$ occurs with odd multiplicity in $V \downarrow_{H}$.

Proof. We use induction on $|G: H|$. By Theorem 9 we may assume that $H$ is not a normal subgroup of $G$. So there exists $H \subsetneq N \subsetneq G$ such that $H$ is subnormal in $N$ and $N$ is normal in $G$. As $|N: H|<|G: H|$, our inductive assumption implies that there is a unique self-dual irreducible $F N$-module $W$ such that $U$ has odd multiplicity in $W \downarrow_{H}$. Let $V$ be the canonical $F G$-module over $W$.

Now $V \downarrow_{N}=W \oplus W_{2} \oplus \cdots \oplus W_{t}$ is the sum of the distinct $G$-conjugates of $W$. As $W$ is self-dual, all the $W_{i}$ are self-dual. By choice of $W, U$ appears with even multiplicity in $W_{i \downarrow_{H}}$, for $i=2, \ldots, t$. Since $V \downarrow_{H}=W \downarrow_{H} \oplus W_{2} \downarrow_{H} \oplus \cdots \oplus W_{t \downarrow_{H}}$, we deduce that $U$ appears with odd multiplicity in $V \downarrow_{H}$.

Now let $V^{\prime}$ be a self-dual irreducible $F G$-module such that $U$ occurs with odd multiplicity in $V^{\prime} \downarrow_{H}$. Write $V^{\prime} \downarrow_{N}=e\left(W_{1}^{\prime} \oplus \cdots \oplus W_{s}^{\prime}\right)$, for some odd integer $e$, where $W_{1}^{\prime}, \ldots, W_{s}^{\prime}$ are distinct irreducible $F N$-modules. We claim that one and hence all $W_{i}^{\prime}$ are self-dual. For suppose otherwise. As $V^{\prime} \downarrow_{N}$ is self-dual, for each $i$ there is a unique $j \neq i$ such that $W_{i}^{\prime *} \cong W_{j}^{\prime}$. Then $U$, being self-dual, occurs with the same multiplicity in $W_{i}^{\prime} \downarrow_{H}$ as in $W_{j}^{\prime} \downarrow_{H}$. So $U$ occurs with even multiplicity in $e\left(W_{i}^{\prime} \oplus W_{j}^{\prime}\right)$, and hence with even multiplicity in $V^{\prime} \downarrow_{H}$. This contradiction proved our claim. So $e=1$ and $V^{\prime}$ is the canonical $F G$-module over each $W_{i}^{\prime}$. Now we may assume that $U$ appears with odd multiplicity in $W_{1}^{\prime} \downarrow_{H}$. So $W \cong W_{1}^{\prime}$, by uniqueness of $W$ over $U$, and then $V^{\prime} \cong V$, by uniqueness of $V$ over $W$.

We thank J. Müller for allowing us to include the following example. In the context of the Corollary it shows that the multiplicity of $U$ in $V \downarrow_{H}$ can be arbitrarily large.

Example: Let $p$ be an odd prime, let $F=\mathrm{GF}(p)$ and let $E=\mathrm{GF}\left(p^{2}\right)$. Considering the additive group of $E$, let $Z$ be the group of scalars in $\mathrm{GL}(E) \cong \mathrm{GL}_{2}(F)$ and let $S$ be a Sylow 2-subgroup of GL(E). We choose an involution $t \in S$, depending on the residue class of $p \bmod 4$, as follows.

If $p \equiv 1(\bmod 4)$, let $C$ be a Sylow 2-subgroup of the multiplicative group $F^{\times}$. Then we may assume that $S=\left\{\left[\begin{array}{ll}a & 0 \\ 0 & b\end{array}\right],\left[\begin{array}{ll}0 & a \\ b & 0\end{array}\right] \mid a, b \in C\right\}$ and we take $t=\left[\begin{array}{ll}0 & 1 \\ 1 & 0\end{array}\right]$.

If $p \equiv 3(\bmod 4)$, we take $t$ to be the Frobenius map $\lambda \mapsto \lambda^{p}$, for all $\lambda \in E$. So $S=C \rtimes\langle t\rangle$, where $C$ is a Sylow 2-subgroup of the multiplicative group $E^{\times}$.

Now let $e_{1}, e_{2} \in E$ be non-trivial, such that $t\left(e_{1}\right)=e_{1}$ and $t\left(e_{2}\right)=-e_{2}$. So $E=$ $F e_{1} \oplus F e_{2}$, as $F$-vector spaces. For our example we take $G=E \rtimes Z S$ and its subgroup $H=F e_{2} \rtimes\langle t\rangle$. Then $H$ is a dihedral group which is subnormal in $G$, for example because it is normal in $E \rtimes(Z \times\langle t\rangle)$, and $G /(E \rtimes Z)$ is nilpotent (as it is a 2-group). 
As $E$ is abelian, each of its irreducible $K$-characters is linear and as $|E|$ is odd, each $K$-character may be regarded a 2-Brauer character. Let $\theta \in \operatorname{Irr}(E)$ have kernel $F e_{2}$. Then $\theta$ has stabilizer $E \rtimes\langle t\rangle$ in $G$, from our descriptions of $Z$ and $S$. Let $\hat{\theta}$ be the unique extension of $\theta$ to a Brauer character of $E \rtimes\langle t\rangle$. Then $\psi:=\hat{\theta} \uparrow G$ is an irreducible Brauer character of $G$ and $\psi \downarrow_{E}$ is the sum of $\psi(1)$ distinct $G$-conjugates of $\theta$.

Let $g \in Z S$ and let $\mu$ be a nontrivial linear character of $F e_{2}$. So $\phi:=\mu \uparrow H$ is an irreducible 2-Brauer character of $H$. Now $Z S$ acts on the 1-dimensional $F$-subspaces of $E$, and $Z\langle t\rangle$ is the stabilizer of the subspace $F e_{1}$ in $Z S$. We set $s=|Z S: Z \times\langle t\rangle|$.

Now $\theta^{g}$ is trivial on $F e_{2}$ if and only if $g \in Z\langle t\rangle$. Consequently $\psi \downarrow_{H}$ contains the trivial Brauer character of $H$ with multiplicity $|Z|=p-1$. Suppose that $g \notin Z\langle t\rangle$. Then $\theta^{g} \downarrow_{F e_{2}}$ is a non-trivial character of $F e_{2}$. So there is a unique $z \in Z$ such that $\theta^{g z} \downarrow_{F e_{2}}=\mu$. It follows from this that $\psi \downarrow_{H}$ contains $\phi$ with multiplicity $s-1$. Finally, $p^{2}-1$ has 2 -part $2 s \geq 8$. So $\psi$ is the canonical character of $G$ lying over $\phi$, with odd multiplicity $s-1 \geq 3$.

\section{IRREDUCIBLE SELF-DUAL MODULES OF NON-QUADRATIC TYPE}

Let $V$ be a non-trivial self-dual irreducible $F G$-module. Then by Lemma $7, V$ affords a non-degenerate $G$-invariant alternating form $B$. Let $Q: V \rightarrow F$ be a quadratic form which polarizes to $B$. This means that $Q\left(\lambda v_{1}\right)=\lambda^{2} Q\left(v_{1}\right)$ and $Q\left(v_{1}+v_{2}\right)=Q\left(v_{1}\right)+$ $B\left(v_{1}, v_{2}\right)+Q\left(v_{2}\right)$, for all $\lambda \in F$ and $v_{1}, v_{2} \in V$. However, contrary to what happens when $\operatorname{char}(F) \neq 2, Q$ is not uniquely determined by $B$. In particular $Q$ need not be $G$-invariant.

On the other hand, for many $F G$-modules each $G$-invariant quadratic form is uniquely determined by its polarization:

Lemma 12. Let $G$ be a finite group and let $F$ be an arbitrary field of characteristic 2 . Suppose that $V$ is an $F G$-module which affords a non-degenerate $G$-invariant alternating bilinear form $B$ but $V$ has no trivial quotient. Then $V$ affords at most one $G$-invariant quadratic form which polarizes to $B$.

Proof. Let $Q_{1}$ and $Q_{2}$ be $G$-invariant quadratic forms on $V$ which polarize to $B$. Then $P:=Q_{1}+Q_{2}$ is a $G$-invariant quadratic form which polarizes to $2 B=0$. Thus $P\left(\lambda v_{1}\right)=$ $\lambda^{2} P\left(v_{1}\right)$, and $P\left(v_{1}+v_{2}\right)=P\left(v_{1}\right)+P\left(v_{2}\right)$, for all $\lambda \in F$ and $v_{1}, v_{2} \in V$.

Set $U:=\{m \in V \mid P(v)=0\}$. Then $U$ is a submodule of $V$. Let $g \in G$ and $v \in V$. Then $g v+v \in U$, as $P(g v+v)=P(g v)+P(v)=0$. So $G$ acts trivially on $V / U$, whence $U=V$ by our hypothesis on $V$. We conclude that $P=0$, or equivalently $Q_{1}=Q_{2}$.

Recall the notion of a canonical irreducible $F G$-module introduced after Theorem 9 .

Proposition 13. Let $W$ be a non-trivial self-dual irreducible FN-module and let $V$ be the canonical self-dual irreducible FG-module lying over $W$. Then $V$ has quadratic type if and only if $W$ has quadratic type.

Proof. We adopt the notation of Theorem 9, So $W$ has a unique self-dual extension $\hat{W}$ to its stabilizer $T$ and $V=\hat{W} \uparrow G$. Also $V \downarrow_{N}$ is the sum of the distinct $G$ conjugates of $W$, each occurring with multiplicity 1 . So we can identify $W$ with an $F$-subspace of $V$. 
Suppose first that $V$ affords a $G$-invariant quadratic form $Q$, and let $B$ be its polarization. So $B$ is a non-degenerate $G$-invariant alternating form on $V$. Let $W^{\perp}=\{v \in V \mid$ $B(v, w)=0$, for all $w \in W\}$. Then $W^{\perp}$ is a submodule of $V \downarrow_{N}$ and $V \downarrow_{N} / W^{\perp} \cong W^{*} \cong W$ as $F N$-modules. As $W$ occurs with multiplicity 1 in $V \downarrow_{N}$, we deduce that $W \cap W^{\perp}=0$. So the restriction $B \downarrow_{W}$ to $W$ is non-degenerate. Moreover the restriction $Q \downarrow_{W}$ of $Q$ to $W$ is an $N$-invariant quadratic form on $W$ which polarizes to $B \downarrow_{W}$. So $W$ has quadratic type.

Conversely, suppose that $W$ affords a non-degenerate $N$-invariant quadratic form $q$, and let $b$ be its polarization. Then $b$ is a non-degenerate $N$-invariant alternating form on $W$. We identify $W$ and $\hat{W}$ as $F$-vector spaces. As $\hat{W}$ is self-dual and irreducible, it affords a $T$-invariant non-zero bilinear form, say $b^{\prime}$. Now all $N$-invariant non-zero bilinear forms on $W$ are scalar multiples of each other, as $W$ is irreducible. So $b$ is a scalar multiple of $b^{\prime}$, and in particular $b$ is $T$-invariant.

For $t \in T$, we define a quadratic form $q^{t}$ on $W$ by setting

$$
q^{t}(w):=q(t w), \quad \text { for all } w \in W .
$$

It is clear that $q^{t}$ is $N$-invariant, and also that $q^{t}$ polarizes to $b$, as $b$ is $T$-invariant. So $q^{t}=q$, according to Lemma 12. This establishes that $q$ is $T$-invariant, and shows that $\hat{W}$ is of quadratic type.

Next, we may decompose $V=\hat{W}^{\uparrow}{ }^{G}$ as $F$-vector space

$$
V=\left(g_{1} \otimes \hat{W}\right) \oplus\left(g_{1} \otimes \hat{W}\right) \oplus \cdots \oplus\left(g_{n} \otimes \hat{W}\right),
$$

where $g_{1}, \ldots, g_{n}$ is a transversal to $T$ in $G$. By a standard procedure, we may define the induced forms $b \uparrow^{G}$ and $q \uparrow^{G}$ on $V$ using

$$
\begin{aligned}
b^{\uparrow}\left(\sum_{i=1}^{n} g_{i} \otimes w_{i}, \sum_{j=1}^{n} g_{j} \otimes x_{j}\right) & =\sum_{i=1}^{n} b\left(w_{i}, x_{i}\right), \\
q^{\uparrow} G\left(\sum_{i=1}^{n} g_{i} \otimes w_{i}\right) & =\sum_{i=1}^{n} q\left(w_{i}\right),
\end{aligned}
$$

for all $w_{i}, x_{j} \in \hat{W}$. Then $b \uparrow^{G}$ is a $G$-invariant alternating bilinear form on $V$ and $q \uparrow^{G}$ is a $G$-invariant quadratic form on $V$ which polarizes to $b \uparrow^{G}$. So $V$ is of quadratic type.

Proposition 14. Let $V$ be a self-dual irreducible FG-module and suppose that some selfdual irreducible $F N$-module $W$ occurs with multiplicity e $>1$ in $V \downarrow_{N}$. Then e is even and $V$ has quadratic type.

Proof. We adopt the notation of Theorem 9. So $W$ has a unique self-dual extension $\hat{W}$ to its stabilizer $T$ and $V=(S \otimes \hat{W}) \uparrow^{G}$, where $S$ is a self-dual irreducible $F(T / N)$-module. Now $W$ occurs with multiplicity 1 in $\left(\hat{W}^{\uparrow} G\right) \downarrow_{N}$. So the multiplicity $e$ of $W$ in $V \downarrow_{N}$ equals $\operatorname{dim}(S)$. As $e>1$, we deduce that $S$ is non-trivial. But then $\operatorname{dim}(S)$ is even, according to Lemma 7. Finally, since $S$ and $\hat{W}$ are both non-trivial and self-dual, $S \otimes \hat{W}$ has quadratic 
type, by the remark below. This in turn implies that $V=(S \otimes \hat{W}) \uparrow G$ has quadratic type.

Remark: Suppose that $U$ and $V$ are $F G$-modules which afford non-degenerate $G$ invariant alternating bilinear forms $B_{U}$ and $B_{V}$, respectively. According to Sin and Willems [SW, Proposition 3.4] there is a quadratic form $Q$ on $U \otimes_{F} V$, which polarizes to $B_{U} \otimes B_{V}$, such that $Q(u \otimes v)=0$, for all $u \in U$ and $v \in V$. These properties uniquely specify $Q$. For, if $u_{1}, \ldots, u_{n}$ and $v_{1}, \ldots, v_{m}$ are bases for $U$ and $V$, respectively, then for all $\lambda_{i j} \in F$

$$
Q\left(\sum \lambda_{i j} u_{i} \otimes v_{j}\right):=\sum \lambda_{i j} \lambda_{i^{\prime} j^{\prime}} B_{U}\left(u_{i}, u_{i^{\prime}}\right) B_{V}\left(v_{j}, v_{j^{\prime}}\right),
$$

where $i, i^{\prime}$ range over $1, \ldots, n$ and $j, j^{\prime}$ over $1, \ldots, m$. Any basic tensor $u \otimes v$ can be written as $\sum \alpha_{i} \beta_{j} u_{i} \otimes v_{j}$, for scalars $\alpha_{i}, \beta_{j}$. Then in the expression for $Q(u \otimes v)$, the term indexed by $(i, j),\left(i^{\prime}, j^{\prime}\right)$ can be cancelled with the term indexed by $\left(i^{\prime}, j\right),\left(i, j^{\prime}\right)$, for $i \neq i^{\prime}$. Likewise pairs of terms with $j \neq j^{\prime}$ cancel. Finally, all terms with $i=i^{\prime}$ or $j \neq j^{\prime}$ are zero as $B_{U}$ and $B_{V}$ are alternating. It is now clear that $Q$ is $G$-invariant.

We turn our attention to those irreducible $F N$-modules that are not self-dual but are $G$-conjugate to their duals. To investigate these, we require a familiar concept.

Suppose that $W$ is an irreducible $F N$-module, with stabilizer $T$ in $G$. Then

$$
T^{*}=\left\{g \in G \mid W^{g} \cong W \text { or } W^{g} \cong W^{*}\right\}
$$

is a subgroup of $G$ containing $T$, called the extended stabilizer of $W$. If $W$ and $W^{*}$ are non-isomorphic and $G$-conjugate, then $\left|T^{*}: T\right|=2$. Otherwise $T=T^{*}$.

Proposition 15. Let $W$ be an irreducible FN-module which is not self-dual. Then all self-dual irreducible FG-modules lying over $W$ are of quadratic type.

Proof. If $W$ is not $G$-conjugate to $W^{*}$, there are no self-dual irreducible $F G$-modules lying over $W$. So we may assume that $W$ is $G$-conjugate to $W^{*}$ and that $\left|T^{*}: T\right|=2$.

Let $V$ be a self-dual irreducible $F G$-module lying over $W$. Then $V=U \uparrow G$, where $U$ is the unique irreducible submodule of $V \downarrow_{T}$ lying over $W$. Likewise $V \cong V^{*}=\left(U^{*}\right) \uparrow G$. So $U^{*}$ is the unique irreducible submodule of $V \downarrow_{T}$ lying over $W^{*}$. Note that $U^{*} \not U$, as $W$ and $W^{*}$ are not $T$-conjugate.

Set $X:=U \uparrow^{T^{*}}$. Then $V=X \uparrow G$, and $X$ is an irreducible submodule of $V \downarrow_{T^{*}}$. Now $U$ is a submodule of $X \downarrow_{T}$. So by uniqueness of $U, X$ is the unique irreducible submodule of $V \downarrow_{T^{*}}$ lying over $W$. Likewise $X^{*}$ is the unique irreducible submodule of $V \downarrow_{T^{*}}$ lying over $W^{*}$. But $X$ lies over $W^{*}$, as $W$ and $W^{*}$ are $T^{*}$-conjugate. So $X \cong X^{*}$.

Let $\tau \in T^{*} \backslash T$. Then $X \downarrow_{T}=U \oplus \tau U$. But $U$ and $U^{*}$ are non-isomorphic irreducible submodules of $X \downarrow_{T}$. So $X \downarrow_{T}=U \oplus U^{*}$, whence $\tau U \cong U^{*}$.

Let $B$ be a $G$-invariant non-degenerate alternating bilinear form on $V$, and let $X^{\perp}$ be the orthogonal complement of $X$ in $V \downarrow_{T^{*}}$ with respect to $B$. Then $X \cong X^{*} \cong V / X^{\perp}$. So $X \cap X^{\perp}=0$, by uniqueness of $X$. This shows that the restriction $B_{X}$ of $B$ to $X$ is a ( $T^{*}$-invariant) non-degenerate alternating bilinear form on $X$. Since $U$ is irreducible but 
not self-dual, it is totally isotropic with respect to $B_{X}$, and likewise, so is $\tau U$. We define $Q: X \rightarrow F$ via

$$
Q\left(u_{1}+\tau u_{2}\right)=B\left(u_{1}, \tau u_{2}\right), \quad \text { for all } u_{1}, u_{2} \in U .
$$

Then $Q$ is a quadratic form which polarizes to $B_{X}$. As $Q$ vanishes on the subspaces $U$ and $\tau U$, it is an example of a hyperbolic form.

We now check that $Q$ is $T^{*}$-invariant. It is certainly $T$-invariant, as $T$ fixes $U$ and $\tau U$, and preserves $B$. Suppose that $\tau^{\prime} \in T^{*} \backslash T$. Then $\tau^{\prime} u_{1} \in \tau U$ and $\tau^{\prime} \tau u_{2} \in U$. So

$$
Q\left(\tau^{\prime}\left(u_{1}+\tau u_{2}\right)\right)=B\left(\tau^{\prime} \tau u_{2}, \tau^{\prime} u_{1}\right)=B\left(\tau u_{2}, u_{1}\right)=B\left(u_{1}, \tau u_{2}\right)=Q\left(u_{1}+\tau u_{2}\right),
$$

since $\tau^{\prime}$ also leaves $B$ invariant. So $Q$ is indeed $T^{*}$-invariant.

Finally, the induced form $Q \uparrow^{G}$ is a $G$-invariant quadratic form on $V$ which polarizes to the $G$-invariant non-degenerate alternating bilinear form $B_{X} \uparrow G$. So $V$ is of quadratic type, as required.

Proof of Theorem [3. Let $V$ be a self-dual irreducible $F G$-module which is not of quadratic type, such that $N$ does not act trivially on $V$. Write $V \downarrow_{N}=e\left(W_{1} \oplus \cdots \oplus W_{t}\right)$, where $e>0$ and $W_{1}, \ldots, W_{t}$ is a $G$-orbit of irreducible $F N$-modules, each of which is irreducible and non-trivial.

It follows from Proposition 15 that each $W_{i}$ is self-dual, and then from Proposition 14 that $e=1$. So $V$ is the canonical self-dual irreducible $F G$-module over each $W_{i}$. Then Proposition 13 implies that each $W_{i}$ has non-quadratic type.

We now show how the techniques we have developed above can be employed to obtain a criterion for all self-dual irreducible $F G$-modules to be of quadratic type.

Corollary 16. Let $N$ be a normal subgroup of $G$. Then all non-trivial self-dual irreducible $F G$-modules are of quadratic type if and only if the same is true of both $F N$ and $F G / N$.

Proof. Suppose first that all non-trivial self-dual irreducible $F G$-modules are of quadratic type. Then the same is obviously true for $G / N$ and we must show that it is true for $N$. Let $W$ be a non-trivial self-dual irreducible $F N$-module and let $V$ be the canonical self-dual irreducible $F G$-module over $W$. Then $V$ is irreducible and of quadratic type, from the hypothesis. So $W$ is of quadratic type, according to Proposition 13 .

Conversely, suppose that all non-trivial self-dual irreducible modules of both $N$ and $G / N$ are of quadratic type. Then for the sake of contradiction, suppose that $V$ is a selfdual irreducible $F G$-module which has non-quadratic type. Theorem 3 implies that $N$ acts trivially on $V$. So $V$ is a self-dual irreducible $F(G / N)$-module of non-quadratic type, contrary to hypothesis.

\section{IRREDUCIBLE SELF-DUAL MODULES OF NON-ABELIAN FINITE SIMPLE GROUPS}

The proof of Corollary [16] shows that in an inductive approach to deciding whether a self-dual irreducible $F G$-module is of quadratic type, the main difficulty lies in solving the problem for non-abelian simple groups, and as far as we know, this is an unsolved and difficult question. See [W, Remark 3.4(a)]. 
At a simpler, but by no means straightforward, level, we can ask if every non-abelian simple group has a non-trivial irreducible module of quadratic type. The answer is no, for according to [HM], the Mathieu simple group $M_{22}$ has no such modules. We were unable to find an explicit reference to the calculations needed to verify this in the literature. So we outline a proof here, which only assumes some knowledge of the irreducible Brauer characters of certain groups.

We use the notation and decomposition matrices from ModAtlas and character tables from GAP. So $M_{22}$ has exactly two non-trivial self-dual irreducible Brauer characters $\phi_{4}$ and $\phi_{7}$, with degrees 34 and 98, respectively.

Now $M_{22}$ has two conjugacy classes of maximal subgroups isomorphic to the alternating group $A_{7}$. The restriction of $\phi_{4}$ to any $A_{7}$ is the sum of an irreducible character $\psi$ of degree 20 plus another of degree 14. In turn, the restriction of $\psi$ to $A_{6}$ is the sum of an irreducible character $\mu$ of degree 4 plus two irreducible characters of degree 8. Examining the values of $\mu$, we see that it is the Brauer character of a representation defined over $\mathbb{F}_{2}$. So $\mu$ cannot be of quadratic type. For the order of $A_{6}$ is greater than the order of each of the two orthogonal groups $O_{+}(4,2) \cong S_{3}>C_{2}$ and $O_{-}(4,2) \cong S_{5}$. It now follows from [GW95, Lemma 1.2] that $\phi_{4}$ is not of quadratic type.

Next we observe that $\phi_{2} \phi_{3}=2 \phi_{1}+\phi_{7}$. Here $\phi_{2}$ and $\phi_{3}=\bar{\phi}_{4}$ are of degree 10, and $\phi_{1}$ is the trivial Brauer character. Now $\phi_{2} \phi_{3}$ is the Brauer character of the ring $E$ of $F$-endomorphisms of a module affording $\phi_{2}$. Let $\operatorname{Tr}: E \rightarrow F$ be the trace map and let $W=\left\{A \in E \mid \operatorname{Tr}(A)=0_{F}\right\}$. Then $W$ is a submodule of $E$ and $E / W$ is the trivial module. So $W$ has Brauer character $\phi_{1}+\phi_{7}$. Clearly the identity map $I \in E$ spans the unique trivial submodule of $W$. Now for each $A \in W$, set $Q(A)$ as the coefficient of $x^{n-2}$ in the $F$-characteristic polynomial of $A$. Then $Q$ is a $G$-invariant quadratic form, with polarization $B(A, B)=\operatorname{Tr}(A B)$, for all $A, B \in W$. In particular $I$ spans $\operatorname{Rad}(Q)$. As $I$ has characteristic polynomial $(x-1)^{10}=x^{10}+x^{8}+x^{2}+1$, we see that $Q(I)=1_{F}$. So the singular radical $\operatorname{Rad}_{0}(Q)$ is 0 . Now [GW95, Theorem 1.3] implies that $\phi_{7}$ is not of quadratic type.

On the other hand, each of the remaining 25 sporadic finite simple groups does have irreducible FG-modules of quadratic type. To show this, we need [F, Corollary IV.11.9]. We give an elementary proof here, for the convenience of the reader:

Lemma 17. Let $G$ be a finite group and let $F$ be a perfect field of characteristic 2 . Then each self-dual but non-quadratic irreducible FG-module is in the principal 2-block of $G$.

Proof. Let $M$ be a self-dual irreducible $F G$-module which is not in the principal 2-block of $G$. We aim to show that $M$ has quadratic type. For this, we exploit the fact that there is no module $W$ such that $\operatorname{soc}(W)$ is the trivial module and $\frac{W}{\operatorname{soc}(W)} \cong M$.

Let $B$ be a $G$-invariant non-degenerate symplectic bilinear form on $M$, and let $Q$ be a quadratic form on $M$ which polarizes to $B$. For all $g \in G$, define $Q^{g}(m):=Q(g m)$. Then $Q^{g}$ is a quadratic form which polarizes to $B$, as

$$
Q^{g}\left(m_{1}+m_{2}\right)=Q\left(g m_{1}\right)+B\left(g m_{1}, g m_{2}\right)+Q\left(g m_{2}\right)=Q^{g}\left(m_{1}\right)+B\left(m_{1}, m_{2}\right)+Q^{g}\left(m_{2}\right) .
$$


Consider the quadratic form $Q+Q^{g}$. This is additive and satisfies $\left(Q+Q^{g}\right)(\lambda m)=$ $\lambda^{2}\left(Q+Q^{g}\right)(m)$, for all $\lambda \in F$ and $m \in M$. As $F$ is perfect, there exists $\phi_{g} \in M^{*}$ such that $Q(\mathrm{gm})=Q(m)+\phi_{g}(m)^{2}$, for all $m \in M$. Now for $g, h \in G$, and $m \in M$ we have

$$
\begin{aligned}
Q(m)+\phi_{g h}(m)^{2} & =Q(g h m) \\
& =Q(h m)+\phi_{g}(h m)^{2} \\
& =Q(m)+\phi_{h}(m)^{2}+h^{-1} \phi_{g}(m)^{2}
\end{aligned}
$$

So $\phi: G \rightarrow M^{*}$ satisfies the cocycle condition $\phi_{g h}=\phi_{h}+h^{-1} \phi_{g}$, for all $g, h \in G$.

Now take $W$ to be the Cartesian product $M \times F$, endowed with the obvious $F$-vector space structure. Define an $F G$-module structure on $W$ via

$$
g(m, \lambda)=\left(g m, \phi_{g}(m)+\lambda\right), \quad \text { for all } m \in M, \lambda \in F \text { and } g \in G .
$$

This is an action because for all $g, h \in G$, we have

$$
\begin{aligned}
(g h)(m, \lambda) & =\left(g h m, \phi_{g h}(m)+\lambda\right) \\
& =\left(g h m, \phi_{g}(h m)+\phi_{h}(m)+\lambda\right) \\
& =g\left(h m, \phi_{h}(m)+\lambda\right) \\
& =g(h(m, \lambda)) .
\end{aligned}
$$

Clearly $W$ has a submodule $0 \times F$ isomorphic to the trivial $F G$-module $F$, and $W$ modulo this submodule is isomorphic to $M$. Our assumption on $M$ forces $W \cong M \oplus F$ as $F G$ modules. So there is $\psi \in M^{*}$ such that $m \mapsto(m, \psi(m))$, for $m \in M$, is an injective $F G$-module map $M \rightarrow W$.

Now on the one hand $g(m, \psi(m))=(g m, \psi(g m))$. On the other hand $g(m, \psi(m))=$ $\left(g m, \phi_{g}(m)+\psi(m)\right)$. Comparing these expressions, we see that $\phi_{g}(m)+\psi(g m)=\psi(m)$, for all $g \in G$. Finally, define the quadratic form $\hat{Q}$ on $M$ via

$$
\hat{Q}(m)=Q(m)+\psi(m)^{2}, \quad \text { for all } m \in M .
$$

Then it is clear that $\hat{Q}$ polarizes to $B$. Furthermore, for all $g \in G$ we have

$$
\hat{Q}(g m)=Q(g m)+\psi(g m)^{2}=Q(m)+\phi_{g}(m)^{2}+\psi(g m)^{2}=Q(m)+\psi(m)^{2}=\hat{Q}(m) .
$$

So $\hat{Q}$ is $G$-invariant.

Using GAP] and [ModAtlas], the only sporadic finite simple groups which do not have a real non-principal 2-block are $M_{11}, M_{22}, M_{23}$ and $M_{24}$. Now $M_{11}$ has an orthogonal irreducible $K$-character $\chi_{2}$, of degree 10 , whose restriction to 2-regular elements is a self-dual irreducible Brauer character $\phi_{2}$. So $\phi_{2}$ has quadratic type. Similarly $M_{24}$ has an orthogonal irreducible $K$-character $\chi_{7}$, of degree 252, whose restriction to 2-regular elements contains the self-dual irreducible Brauer character $\phi_{6}$ with multiplicity 1 , but does not contain the trivial Brauer character. So $\phi_{6}$ has quadratic type. Finally, $\phi_{6}$ restricts to an irreducible Brauer character of a maximal subgroup $M_{23}$. So $M_{23}$ also has a quadratic type irreducible Brauer character. 
All other simple group whose modular representations are tabulated in the modular Atlas have quadratic type irreducible Brauer characters, and we suspect that $M_{22}$ may be unique among all non-abelian finite simple groups in not having such a character. We note that the automorphism group of $M_{22}$ does have irreducible modules of quadratic type, since Proposition 15 applies to certain irreducible modules of the automorphism group that are induced from irreducible modules of $M_{22}$ that are not self-dual.

\section{REAL WEAKLY REGULAR 2-BLOCKS}

We continue to assume that $G$ is a finite group and $N$ is a normal subgroup of $G$. The results in this section include real refinements of [M, Theorem 4.4, Corollary 4.5]. If $C$ is a conjugacy class of $G$, then $C^{+}$is the sum of its elements in $R G$. Also $C^{o}$ is the class consisting of the inverses of the elements of $C$. Each $z \in \mathrm{Z}(F G)$ can be written $z=\sum \beta(z, C) C^{+}$, where $C$ ranges over the conjugacy classes of $G$ and $\beta(z, C) \in F$.

We use standard notation and results on blocks. In particular, corresponding to each 2block $B$ of $G$, there is a primitive idempotent $e_{B}$ of the centre $\mathrm{Z}(F G)$ of $F G$, an $F$-algebra homomorphism $\omega_{B}: \mathrm{Z}(F G) \rightarrow F$, called the central character of $B$, and a 2-subgroup $D$ of $G$ called a defect group of $B$. Then $D$ is only determined up to $G$-conjugacy, and $|D|=2^{d}$, where $d \geq 0$ is called the defect of $B$. We use $\operatorname{Irr}(B)$ and $\operatorname{IBr}(B)$ to denote the irreducible $K$-characters and irreducible Brauer characters in $B$, respectively.

Let $\chi \in \operatorname{Irr}(B)$, let $\psi$ be an irreducible constituent of $\chi \downarrow_{N}$ and let $b$ be the 2-block of $N$ containing $\psi$. Then $B$ is said to cover $b$, and the 2-blocks of $N$ covered by $B$ form a single $G$-orbit. Set $e_{b}^{G}$ as the sum of the distinct $G$-conjugates of $e_{b}$. Then $e_{b}^{G}$ is an idempotent in $\mathrm{Z}(F G)$ which is the sum of the block idempotents of all blocks of $G$ which cover $b$.

Recall that $B$ is said to be weakly regular (with respect to $N$ ) if it has maximal defect among the set of blocks of $G$ which cover $b$. This happens if and only if $B$ has a defect group $D$ such that $D N / N$ is a Sylow 2-subgroup of the stabilizer of $b$ in $G$.

Let $\chi$ be a $K$-character or Brauer character belonging to $B$. Then $\chi(1)_{2} \geq|G: D|_{2}$. If equality occurs, we say that $\chi$ has height 0 . Recall that if $\chi$ is irreducible, its central character is defined by $\omega_{\chi}\left(C^{+}\right):=\chi\left(C^{+}\right) / \chi(1)$, for all conjugacy classes $C$ of $G$. It is classical that $\omega_{\chi}\left(C^{+}\right) \in R$. Indeed its image $\omega_{\chi}\left(C^{+}\right)^{*}$ in $F$ is independent of $\chi \in \operatorname{Irr}(B)$, as it equals $\omega_{B}\left(C^{+}\right)$. Suppose now that $\theta \in \operatorname{IBr}(B)$ has height 0 . We claim that for all 2-regular conjugacy classes $C$ of $G$

$$
\frac{\theta\left(C^{+}\right)}{\theta(1)} \in R \quad \text { and } \quad\left(\frac{\theta\left(C^{+}\right)}{\theta(1)}\right)^{*}=\omega_{B}\left(C^{+}\right) .
$$

For, it is known that there are integers $n_{\chi}$ such that $\theta \equiv \sum_{\chi \in \operatorname{Irr}(B)} n_{\chi} \chi$ on the 2-regular elements of $G$. As $\chi\left(C^{+}\right) / \chi(1)$ and $\chi(1) / \theta(1)$ belong to $R$, we get

$$
\frac{\theta\left(C^{+}\right)}{\theta(1)}=\sum_{\chi \in \operatorname{Irr}(B)}\left(\frac{\chi\left(C^{+}\right)}{\chi(1)}\right)\left(\frac{n_{\chi} \chi(1)}{\theta(1)}\right) \quad \text { belongs to } R \text {. }
$$

Moreover $\left(\frac{\theta\left(C^{+}\right)}{\theta(1)}\right)^{*}=\omega_{B}\left(C^{+}\right)\left(\frac{\sum_{\chi \in \operatorname{Irr}(B)} n_{\chi} \chi(1)}{\theta(1)}\right)^{*}=\omega_{B}\left(C^{+}\right)$. 
Our first result includes a proof of part (i) of Theorem 4:

Lemma 18. Let $b$ be a 2-block of $N$. Then $G$ has an odd number of weakly regular 2 -blocks covering $b$. So $G$ has a real weakly regular 2 -block covering $b$ if and only if $b$ is $G$-conjugate to $b^{o}$.

Let $B$ be a weakly regular 2-block covering b. Then $\beta\left(e_{B}, C\right) \omega_{B}\left(C^{+}\right)=\beta\left(e_{b}^{G}, C\right) \omega_{b}\left(C^{+}\right)$, for all conjugacy class $C$ of $G$ contained in $N$.

Proof. The first statement is proved in Lemma 5.1 of [GM], so we merely summarize the argument here. There is a defect preserving bijection between the blocks of $G$ covering $b$ and the blocks of the $G$-stabilizer of $b$ covering $b$. So we may assume that $b$ is $G$-invariant.

Let $B$ be as in the statement. In particular $e_{B}=e_{B} e_{b}$. So $1_{F}=\omega_{B}\left(e_{B}\right)=\omega_{B}\left(e_{b}\right)$. Thus there is a conjugacy class $L$ of $G$ contained in $N$ such that $\beta\left(e_{b}, L\right) \omega_{B}\left(L^{+}\right) \neq 0_{F}$. Now $L$ is 2-regular, as it is in the support of the block idempotent $e_{b}$. As $e_{b}$ is a sum of block idempotents of blocks of $G$ with a defect group contained in $D, L$ has a defect group contained in $D$. But $\omega_{B}\left(L^{+}\right) \neq 0_{F}$. So $L$ has a defect group containing the defect group $D$ of $B$. We deduce that $D$ is a defect group of $L$.

Corollary 3.2 of GM] implies that $\beta\left(e_{B}, L\right)=\omega_{B}\left(L^{o+}\right)$. But $\omega_{B}\left(L^{o+}\right)=\omega_{B^{\prime}}\left(L^{o+}\right)$, for each block $B^{\prime}$ of $G$ which covers $b$, as $L \subseteq N$. So, again by Corollary 3.2 of GM] $\beta\left(e_{B}, L\right)=\beta\left(e_{B^{\prime}}, L\right)$, if $B^{\prime}$ is in addition weakly regular. On the other hand $\beta\left(e_{B^{\prime}}, L\right)=0_{F}$, if $B^{\prime}$ is not weakly regular. As $e_{b}$ is the sum of the block idempotents of all blocks of $G$ covering $b$, we see that $\beta\left(e_{b}, L\right)=\beta\left(e_{B}, L\right) \rho$, where $\rho$ is the number of weakly regular 2-blocks of $G$ covering $b$. It follows from this that $\rho$ is odd.

Suppose that there is a real weakly regular 2 -block $B$ of $G$ which covers $b$. Then $B=B^{o}$ also covers $b^{o}$. So $b$ is $G$-conjugate to $b^{o}$. Conversely, suppose that $b$ is $G$-conjugate to $b^{o}$. Then taking contragredients of blocks is an involution on the set of weakly regular 2-block of $G$ covering $b$. As this set has odd size $\rho$, we deduce that there is a real weakly regular 2-block of $G$ which covers $b$.

For the last statement, let $C$ be a conjugacy class of $G$ which is contained in $N$ for which $\beta\left(e_{B}, C\right) \omega_{B}\left(C^{+}\right) \neq 0_{F}$ or $\beta\left(e_{b}, C\right) \omega_{b}\left(C^{+}\right) \neq 0_{F}$. As $\omega_{B}\left(C^{+}\right)=\omega_{b}\left(C^{+}\right)$, the argument above implies that $D$ is a defect group of $C$. But then $\beta\left(e_{b}, C\right)=\beta\left(e_{B}, C\right) \rho=\beta\left(e_{B}, C\right)$, as $\operatorname{char}(F)=2$. We conclude that $\beta\left(e_{B}, C\right) \omega_{B}\left(C^{+}\right)=\beta\left(e_{b}, C\right) \omega_{b}\left(C^{+}\right)$.

We need one more result before proving part (ii) of Theorem 4 ;

Lemma 19. Let $b$ be a real $G$-invariant 2-block of $N$. Then $G$ has a self-dual Brauer character $\phi$ such that $\phi$ vanishes off $N$ and $\phi_{\downarrow_{N}}=e\left(\theta_{1}+\cdots+\theta_{t}\right)$ where both $e$ and $t$ are odd and $\theta_{1}, \ldots, \theta_{t}$ are distinct self-dual height 0 irreducible Brauer characters in $b$.

Proof. Note that we are not claiming that $\phi$ is irreducible.

Consider the $G$-set $X:=\left\{\theta \in \operatorname{IBr}(b) \mid \theta\right.$ has height zero and $\left.\Phi_{\theta}(1)_{2}=|N|_{2}\right\}$. Then $|X|$ is odd, according to Lemma 5. Also duality is an involution on $X$. So there is a $G$-orbit $\theta_{1}, \ldots, \theta_{t}$ in $X$, with $t$ odd and all $\theta_{i}$ self-dual and of height 0 . 
Let $T$ be the inertial group of $\theta_{1}$ in $G$. Then $T$ contains a Sylow 2-subgroup $S$ of $G$. As $S N / N$ is a 2-group, $\theta_{1}$ has a unique extension $\hat{\theta}_{1}$ to an irreducible Brauer character of $S N$. Notice that $\hat{\theta}_{1}$ vanishes off $N$, as $N$ contains all 2-regular elements of $S N$.

Set $\phi:=\hat{\theta}_{1} \uparrow G$. Then $\phi$ is self-dual and $\phi \downarrow_{N}=\frac{1}{[S N: N]}\left(\theta_{1} \uparrow G\right) \downarrow_{N}=e\left(\theta_{1}+\cdots+\theta_{t}\right)$, where $e=[T: S N]$ is odd. Finally $\phi$ vanishes off $N$ as $\hat{\theta}_{1}$ vanishes off $N$.

We now prove the uniqueness part (ii) of Theorem 4:

Lemma 20. Let b be a real 2-block of $N$. Then $G$ has a unique real 2-block which covers $b$ and which is weakly regular with respect to $N$.

Proof. We may assume that $b$ is $G$-invariant, and we let $B$ be any real weakly regular 2-block of $G$ covering $b$. Let $\phi$ be the Brauer character of $G$ defined in Lemma 19. So $\phi \downarrow_{N}=e\left(\theta_{1} \cdots+\theta_{t}\right)$ where et is odd and $\theta_{1}, \ldots, \theta_{t}$ are distinct self-dual height 0 irreducible Brauer characters in $b$. Write $\phi=\sum_{\mu \in \operatorname{IBr}(G)} m_{\mu} \mu$, where $m_{\mu}$ are non-negative integers. Then $\phi_{B}:=\sum_{\mu \in \operatorname{IBr}(B)} m_{\mu} \mu$ is the $B$-part of $\phi$.

Let $C$ be a 2-regular conjugacy class of $G$ which is contained in $N$. Then $\theta_{i}\left(C^{+}\right)=$ $\theta_{1}\left(C^{+}\right)$, for $i=1, \ldots, t$, as $\theta_{i}$ is $G$-conjugate to $\theta_{1}$. So

$$
\left(\frac{\phi\left(C^{+}\right)}{\theta_{1}(1)}\right)^{*}=\left(\frac{e t \theta_{1}\left(C^{+}\right)}{\theta_{1}(1)}\right)^{*}=\omega_{b}\left(C^{+}\right)=\omega_{B}\left(C^{+}\right)
$$

where we have used (5).

Next let $\hat{e_{B}}$ be the unique idempotent in $\mathrm{Z}(R G)$ with $\hat{e_{B}}{ }^{*}=e_{B}$. Then for all $\mu \in \operatorname{IBr}(G)$ we have $\mu\left(\hat{e_{B}}\right)=\mu(1)$ or $0_{R}$, as $\mu$ does or does not belong to $B$, respectively. So

$$
\left(\frac{\phi_{B}(1)}{\theta_{1}(1)}\right)^{*}=\left(\frac{\phi\left(\hat{e_{B}}\right)}{\theta_{1}(1)}\right)^{*}=\sum \beta\left(e_{B}, C^{+}\right) \omega_{B}\left(C^{+}\right)=\sum \beta\left(e_{b}, C^{+}\right) \omega_{b}\left(C^{+}\right)=\omega_{b}\left(e_{b}\right)=1_{F} .
$$

Here in both sums, $C$ ranges over the conjugacy classes of $G$ which are contained in $N$, as $\phi$ vanishes off $N$. Also the middle equality arises from the last assertion in Lemma 18 .

Now for each $\mu \in \operatorname{IBr}(B)$ with $m_{\mu} \neq 0$, we have $\mu \downarrow_{N}=e_{\mu}\left(\theta_{1} \cdots+\theta_{t}\right)$, for some integer $e_{\mu}>0$. Then by the previous displayed equation

$$
\frac{\phi_{B}(1)}{\theta_{1}(1)}=t \sum_{\mu \in \operatorname{IBr}(B)} m_{\mu} e_{\mu} \quad \text { is an odd integer. }
$$

As $m_{\mu} e_{\mu}=m_{\bar{\mu}} e_{\bar{\mu}}$, it follows that there is a self-dual $\mu \in \operatorname{IBr}(B)$ such that $m_{\mu} e_{\mu}$ is odd. Then $\mu$ is the canonical irreducible Brauer character of $G$ lying over $\theta_{1}$ given by Theorem 2. As $\theta_{1}$ determines $\mu$, which in turn determines $B$, we conclude that $B$ is the only real weakly regular 2 -block of $G$ which covers $b$, as we wished to show.

\section{REFERENCES}

[Atlas] C. Jansen, K. Lux, R. Parker, R. Wilson, An atlas of Brauer characters. Lond. Math. Soc. Mono. New Series, 11. Oxford Science Publications. The Clarendon Press, Oxford University Press, New York, 1995. 
[F] W. Feit, The representation theory of finite groups, North-Holland 1982.

[GAP] The GAP Group, GAP - Groups, Algorithms, and Programming, Version 4.11.0; 2020 , https://www.gap-system.org

[G] R. Gow, Real valued and 2-rational group characters, J. Algebra 61 (1979) 388-413.

[GM] R. Gow, J. Murray, Real 2-regular classes and 2-blocks, J. Algebra 230 (2) (2000) 455-473.

[GW93] R. Gow, W. Willems, Quadratic geometries, projective modules and idempotents, J. Algebra 160 (1993) 257-272.

[GW95] R. Gow, W. Willems, Methods to decide if simple self-dual modules over fields of characteristic 2 are of quadratic type, J. Algebra 175 (1995) 1067-1081.

[Gr] J. A. Green, On the indecomposable representations of a finite group, Math. Zeit. 70 (1959) 430-445.

[HM] G. Hiss, G. Malle, Low-dimensional representations of quasi-simple groups, Lond. Math. Soc. J. Comput. Math. 4 (2001) 22-63.

[KOW] M. Kiyota, T. Okuyama, T. Wada, The heights of irreducible Brauer characters in 2-blocks of the symmetric groups, J. Algebra 368 (2012) 329-344.

[M] M. Murai, Block induction, normal subgroups and characters of height zero, Osaka J. Math. 31 (1994) 9-25.

[ModAtlas] The Modular Atlas homepage. (2018). http://www.math.rwth-aachen.de/ MOC/

[NT] H. Nagao, Y. Tsushima, Representations of finite groups, Academic Press, Inc. , 1989.

[N] G. Navarro, Characters and blocks of finite groups, London Math. Soc. Lecture Notes Series 250 , Cambridge Univ. Press (1998), 287pp.

[R] I. M. Richards, Characters of groups with quotients of odd order, J. Algebra 96 (1985) 45-47.

[SW] P. Sin, W. Willems, G-invariant quadratic forms, J. reine angew. Math. 420 (1991) 45-59.

[W] Wolfgang Willems, Duality and forms in representation theory, Representation theory of finite groups and finite-dimensional algebras (Bielefeld, 1991), Progr. Math., 95, Birkhäuser, Basel, 1991, 509520 .

School of Mathematical Sciences,, University College Dublin, IRELAND

Email address: Rod.Gow@ucd.ie

Department of Mathematics and Statistics, Maynooth University, IRELAND

Email address: John. Murray@mu.ie 